\title{
Stress Intensity Factors for Cracked Finite Plates with Mixed Boundary Condition
}

\author{
Zheyuan Hu, Zheming Zhu, Ruoqi Feng, and Rong Hu \\ Key Laboratory of Energy Engineering Safety and Disaster Mechanics, Ministry of Education, College of Architecture and Environment, \\ Sichuan University, Chengdu 610065, China
}

Correspondence should be addressed to Zheming Zhu; zhemingzhu@hotmail.com

Received 9 July 2013; Accepted 31 July 2013

Academic Editors: k. Mekheimer, A. Z. Sahin, and G.-J. Wang

Copyright (C) 2013 Zheyuan Hu et al. This is an open access article distributed under the Creative Commons Attribution License, which permits unrestricted use, distribution, and reproduction in any medium, provided the original work is properly cited.

\begin{abstract}
The mixed boundary problems for finite plates with one crack or two collinear cracks are studied. Complex stress functions that satisfy the equilibrium equations and compatibility conditions in the cracked plate as well as the stress condition on crack surfaces are presented. Four models, that is, a square plate with one crack or with two collinear cracks and an airfoil-shaped plate with one crack or with two collinear cracks, are established. The unknown coefficients of the complex stress functions are determined by using boundary collocation method (BCM). The effects of crack orientation, crack distance, and boundary condition on SIFs are investigated by combining with BCM, and the corresponding photoelastic experiments are conducted. The test results generally agree with the BCM calculation results.
\end{abstract}

\section{Introduction}

Finite plates with fixed and loaded combination boundary are frequently encountered in engineering practice, such as cantilever beams and aircraft airfoils. In both cases, part of the structures is fixed and the upper face of the cantilever or the windward side of the airfoil will be subjected to load. If such structures contain a crack or multicracks, how to evaluate their stability and strength is a significant subject because the strength of cracked structures is immensely dependent on the geometrical configuration, loading condition, and the crack behavior. In order to precisely predict the stability of the structures of cracked finite plates with mixed boundary condition, it is imperative to implement theoretical and experimental studies so as to obtain the dominative parameters that control the structure stability and, furthermore, to predict engineering disasters.

Varied solutions to stress intensity factors for crack problems are generally in three different ways, namely, analytic, numerical, and experimental methods. For many plane problems, the SIFs can be found in the handbook $[1,2]$. Muskhelishvili [3] established the fundamental equations in terms of complex functions to solve many plane problems. Jing [4] reviewed the numerical methods in literature and listed many available numerical methods, outstanding issues and potential future developments in this field. Marakami [5] used the finite element method to determine the stress intensity factors for a plate with single crack. Zhu et al. [6-11] investigated the effect of crack orientations on SIFs for cracks subjected to uniform load and proposed a brief formula of SIF based on BCM calculation results. Yavuz et al. [12] analyzed the interaction of multiple crack configuration using integral equations derived from crack opening displacement and calculated the SIFs of branched crack and kinked crack. Chen et al. [13] solved a number of multiple crack problems in integral equation approach. Zhu [14] formulated the analytic solution for infinite plate with two collinear cracks and proposed a new fracture criterion for collinear cracks under compression. Wang et al. [15] used BCM to investigate the SIFs of finite rectangle plate in bending, and the results agreed very well with existing literature. Jin et al. [16] studied the effect of confining stress, friction coefficient, crack orientation, and crack distance on SIFs in a finite plate with collinear cracks. Although the crack problem has been widely studied by the above researchers, the issue of finite cracked plate with mixed boundary conditions, which is often encountered in engineering practice, has received limited attention. 


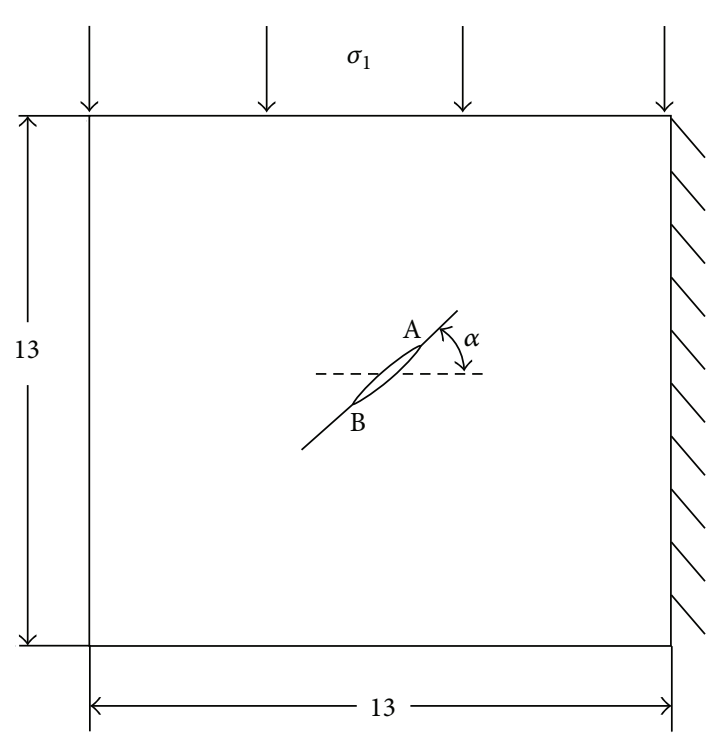

(a) A single crack model

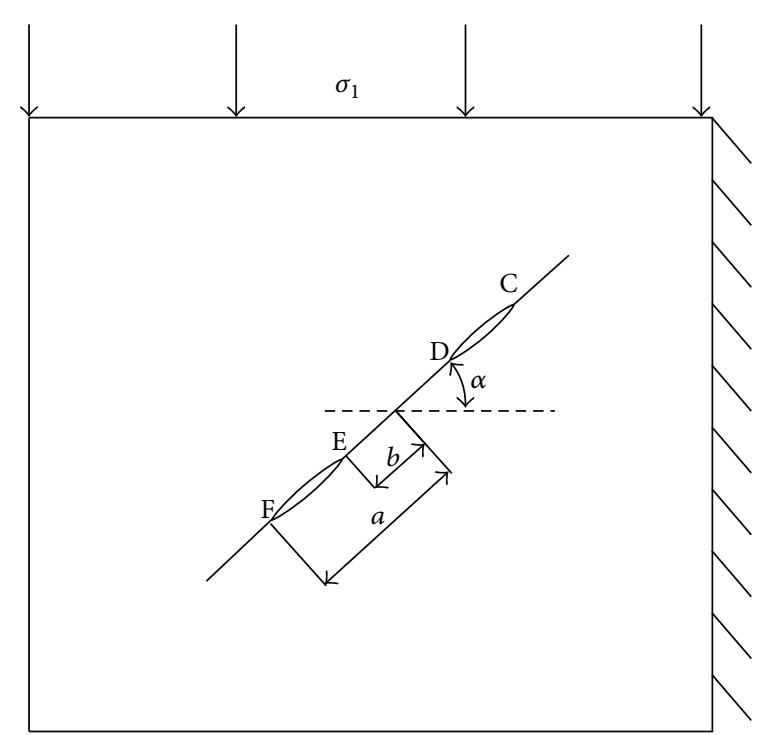

(b) Two collinear cracks model

FIGURE 1: A square plate with (a) a single crack and (b) two collinear cracks under mixed boundary condition.

The objective of this paper is to present the SIF solutions to two kinds of finite cracked plates, a square plate and an airfoil-shaped plate, with mixed boundary conditions and to investigate the effect of crack orientation, the distance between two collinear cracks and boundary condition on SIFs. A set of equations is formulated in terms of complex functions to describe the stress distribution. These stress functions automatically satisfy the equilibrium and compatibility requirements, single-valued displacement condition as well as the stress condition on crack surfaces. Only the boundary conditions on the outer boundary need to be satisfied, and this is achieved by using boundary collocation method (BCM), which is a simple and accurate numerical method and has been well established by many researchers $[6-9,15,17]$.

\section{Complex Stress Functions}

The general solutions to linear elasticity problems, expressed in terms of complex stress potentials $\varphi(z)$ and $\omega(z)$, were first presented by Muskhelishvili [3], and the stresses and displacements of plane problems can be formulated as

$$
\begin{gathered}
\sigma_{x}+\sigma_{y}=2[\Phi(z)+\overline{\Phi(z)}], \\
\sigma_{y}-i \tau_{x y}=\Phi(z)+\Omega(\bar{z})+(z-\bar{z}) \overline{\Phi^{\prime}(z)}, \\
2 G(u+i v)=\kappa \varphi(z)-\omega(\bar{z})-(z-\bar{z}) \overline{\Phi(z)},
\end{gathered}
$$

where $z=x+i y, \bar{z}=x-i y, \Phi(z)=\varphi^{\prime}(z), \Omega(z)=\omega^{\prime}(z)$, $\kappa=(3-v) /(1+v)$ for plane stress problem and $\kappa=1-4 v$ for plane strain problem; $G=E / 2(1+v)$, where $v$ is Poisson's ratio and $E$ is Young's modulus.
Automatically, the equilibrium equations and compatibility conditions are satisfied by these functions. Specific expressions for each stress and displacement component can be obtained if the single-valued displacement condition and boundary conditions (stress and displacement boundary conditions) are satisfied. The single-valued displacement condition can be written as

$$
\kappa \oint \Phi(z)-\oint \Omega(\bar{z})=0
$$

In this paper, a part of the boundary is fixed as shown in Figure 1, so the displacement boundary condition can be expressed as

$$
\kappa \varphi(z)-\omega(\bar{z})-(z-\bar{z}) \overline{\Phi(z)}=0
$$

The resultant force boundary condition which is more accurate than stress boundary condition can be written as

$$
\varphi(z)+\omega(\bar{z})+(z-\bar{z}) \overline{\Phi(z)}=i \int_{z_{0}}^{z}\left(X_{n}+i Y_{n}\right) d s,
$$

where $z_{0}$ is an randomly selected point on the outer boundary.

The plates as shown in Figure 1 are subjected to both stress and displacement boundary conditions (i.e., upper edge loaded with uniform compression and right edge assigned with zero displacements) with one centrally located crack and two collinear cracks, respectively. The crack is designed to coincide with the $x$-axis, which is at an angle $\alpha$ with respect 
to the horizontal $x$-axis. According to Zhu [14], the complex stress functions $\varphi(z), \omega(z)$, and $\Phi(z)$ can be written as

$$
\begin{aligned}
\varphi(z)= & \sqrt{\left(z^{2}-a^{2}\right)\left(z^{2}-b^{2}\right)} \\
& \times \sum_{n=1}^{M} E_{n} z^{n-2}+\sum_{n=1}^{M} F_{n} z^{n}-\frac{1}{2} \sigma z+\frac{1}{2} i \tau z, \\
\omega(z)= & \sqrt{\left(z^{2}-a^{2}\right)\left(z^{2}-b^{2}\right)} \\
& \times \sum_{n=1}^{M} E_{n} z^{n-2}-\sum_{n=1}^{M} F_{n} z^{n}-\frac{1}{2} \sigma z+\frac{1}{2} i \tau z, \\
\Phi(z)= & \frac{1}{\sqrt{\left(z^{2}-a^{2}\right)\left(z^{2}-b^{2}\right)}} \\
& \times \sum_{n=1}^{M} E_{n}\left[n z^{n+1}-(n-1)\left(a^{2}+b^{2}\right) z^{n-1}\right. \\
& +\sum_{n=1}^{M} F_{n} n z^{n-1}-\frac{1}{2} \sigma+\frac{1}{2} i \tau,
\end{aligned}
$$

where $\sigma$ and $\tau$ are normal and shear stresses acting on crack surface, $E_{n}$ and $F_{n}$ are complex constants in general, and $M$ is the number of summation term. The resultant force and displacement boundary can be expressed as

$$
\begin{aligned}
i \int_{z_{0}}^{z}\left(X_{n}+i Y_{n}\right) d s & =\varphi(z)+\omega(z)+(z-\bar{z}) \overline{\Phi(z)}=Q(z), \\
2 G(u+i v) & =\kappa \varphi(z)-\omega(\bar{z})-(z-\bar{z}) \overline{\Phi(z)} \\
& =(\kappa+1) \varphi(z)-Q(z)=T(z) .
\end{aligned}
$$

Inserting (5) into (6), one can have

$$
\begin{aligned}
& \sum_{n=1}^{N} E_{n}\left[\sqrt{\left(z^{2}-a^{2}\right)\left(z^{2}-b^{2}\right)} z^{n-2}\right. \\
& \left.+\sqrt{\left(\bar{z}^{2}-a^{2}\right)\left(\bar{z}^{2}-b^{2}\right)} \bar{z}^{n-2}\right] \\
& +\sum_{n=1}^{N} F_{n}\left(z^{n}-\bar{z}^{n}\right)+(z-\bar{z}) \\
& \text { - conjg }\left\{\frac{1}{\sqrt{\left(z^{2}-a^{2}\right)\left(z^{2}-b^{2}\right)}}\right. \\
& \times \sum_{n=1}^{N} E_{n}\left[n z^{n+1}-(n-1)\left(a^{2}+b^{2}\right) z^{n-1}\right. \\
& \left.\left.+(n-2) a^{2} b^{2} z^{n-3}\right]+\sum_{n=1}^{N} F_{n} n z^{n-1}\right\} \\
& -\sigma z+i \tau \bar{z}=i \int_{z_{0}}^{z}\left(X_{n}+i Y_{n}\right) d s .
\end{aligned}
$$

Let $z=r e^{i \theta}, z-a=r_{1} e^{i \theta 1}, z+a=r_{2} e^{i \theta 2}, z-b=r_{3} e^{i \theta 3}, z+b=$ $r_{4} e^{i \theta 4}$, then

$$
\sqrt{\left(z^{2}-a^{2}\right)\left(z^{2}-b^{2}\right)}=\sqrt{r_{1} r_{2} r_{3} r_{4}} e^{i(\theta 1+\theta 2+\theta 3+\theta 4) / 2}=\rho e^{i \alpha},
$$

where $r$ and $\theta$ are the modulus and angle of a complex. Substituting (9) into (8), the real and imaginary parts of (8) can be separately expressed as

$$
\begin{aligned}
\operatorname{Re}[Q(z)]= & \sum_{k=1}^{M}\left[S_{1 k}+S_{6 k}\right] \operatorname{Re}\left(E_{k}\right) \\
& +\sum_{k=1}^{M}\left[S_{5 k}\right] \operatorname{Im}\left(E_{k}\right)+\sum_{k=1}^{M}\left[S_{4 k}\right] \operatorname{Re}\left(F_{k}\right) \\
& +\sum_{k=1}^{M}\left[-S_{2 k}+S_{3 k}\right] \operatorname{Im}\left(F_{k}\right)-\sigma r \cos \theta+\tau r \sin \theta
\end{aligned}
$$

$$
\begin{aligned}
\operatorname{Im}[Q(z)]= & \sum_{k=1}^{M}\left[S_{5 k}\right] \operatorname{Re}\left(E_{k}\right) \\
& +\sum_{k=1}^{M}\left[S_{1 k}-S_{6 k}\right] \operatorname{Im}\left(E_{k}\right)+\sum_{k=1}^{M}\left[S_{2 k}+S_{3 k}\right] \operatorname{Re}\left(F_{k}\right) \\
& +\sum_{k=1}^{M}\left[-S_{4 k}\right] \operatorname{Im}\left(F_{k}\right)-\sigma r \sin \theta+\tau r \cos \theta .
\end{aligned}
$$

Following the same procedure, the real and imaginary parts of (7) can be separately written as

$$
\begin{aligned}
\operatorname{Re}[T(z)]= & \sum_{k=1}^{M}\left[U_{1 k}-S_{1 k}-S_{6 k}\right] \operatorname{Re}\left(E_{k}\right) \\
& +\sum_{k=1}^{M}\left[-U_{2 k}-S_{5 k}\right] \operatorname{Im}\left(E_{k}\right) \\
& +\sum_{k=1}^{M}\left[U_{3 k}-S_{4 k}\right] \operatorname{Re}\left(F_{k}\right) \\
& +\sum_{k=1}^{M}\left[-U_{4 k}+S_{2 k}-S_{3 k}\right] \operatorname{Im}\left(F_{k}\right) \\
& +\frac{1}{2} \sigma r(1-\kappa) \cos \theta-\frac{1}{2} \tau r(3+\kappa) \sin \theta,
\end{aligned}
$$




$$
\begin{aligned}
\operatorname{Im}[T(z)]= & \sum_{k=1}^{M}\left[U_{2 k}-S_{5 k}\right] \operatorname{Re}\left(E_{k}\right) \\
& +\sum_{k=1}^{M}\left[U_{1 k}-S_{1 k}+S_{6 k}\right] \operatorname{Im}\left(E_{k}\right) \\
& +\sum_{k=1}^{M}\left[U_{4 k}-S_{2 k}-S_{3 k}\right] \operatorname{Re}\left(F_{k}\right) \\
& +\sum_{k=1}^{M}\left[U_{3 k}+S_{4 k}\right] \operatorname{Im}\left(F_{k}\right) \\
& +\frac{1}{2} \sigma r(1-\kappa) \sin \theta-\frac{1}{2} \tau r(1-\kappa) \cos \theta
\end{aligned}
$$

where

$$
\begin{aligned}
& S_{1 k}=2 \rho r^{k-2} \cos [(k-2)+\alpha], \\
& S_{2 k}=2 r^{k} \sin k \theta \text {, } \\
& S_{3 k}=2 k r^{k} \sin \theta \cos (k-1) \theta, \\
& S_{4 k}=2 k r^{k} \sin \theta \sin (k-1) \theta, \\
& S_{5 k}=2 k r^{k+2} \sin \theta \cos \frac{[(k+1) \theta-\alpha]}{\rho} \\
& -\frac{2(k-1)\left(a^{2}+b^{2}\right) \sin \theta \cos [(k-1) \theta-\alpha]}{\rho} \\
& +\frac{2(k-2) a^{2} b^{2} r^{k-2} \sin \theta \cos [(k-3)-\alpha]}{\rho}, \\
& S_{6 k}=2 k r^{k+2} \sin \theta \sin \frac{[(k+1) \theta-\alpha]}{\rho} \\
& -\frac{2(k-1)\left(a^{2}+b^{2}\right) \sin \theta \sin [(k-1) \theta-\alpha]}{\rho} \\
& +\frac{2(k-2) a^{2} b^{2} r^{k-2} \sin \theta \sin [(k-3)-\alpha]}{\rho}, \\
& U_{1 k}=(\kappa+1) \rho r^{k-2} \cos [(k-2)+\alpha], \\
& U_{2 k}=(\kappa+1) \rho r^{k-2} \sin [(k-2)+\alpha] \text {, } \\
& U_{3 k}=(\kappa+1) r^{k} \cos k \theta \text {, } \\
& U_{4 k}=(\kappa+1) r^{k} \sin k \theta \text {. }
\end{aligned}
$$

The crack tip SIFs are [13]

$$
K_{\mathrm{I}}-i K_{\mathrm{II}}=\lim _{z \rightarrow \pm a} 2 \sqrt{2 \pi e^{-i \beta}(z \mp a)} \Phi(z),
$$

where $\beta$ is the crack angle with respect to the positive $x$-axis, and for the right crack tip $\beta=0$, whereas for the left one, $\beta=\pi$. The dimensionless SIFs $Y_{\mathrm{I}}$ and $Y_{\mathrm{II}}$ for the right crack tips are

$$
Y_{\mathrm{I}}-i Y_{\mathrm{II}}=\frac{\left(K_{\mathrm{I}}-i K_{\mathrm{II}}\right)}{K_{0}}=\frac{\sum_{k=1}^{M} E_{k} 2 \sqrt{(a+b)} a^{k-1.5}}{\sigma_{1}},
$$

and for the left crack tips they are

$$
Y_{\mathrm{I}}-i Y_{\mathrm{II}}=\frac{\left(K_{\mathrm{I}}-i K_{\mathrm{II}}\right)}{K_{0}}=\frac{\sum_{k=1}^{M} E_{k} 2 \sqrt{(a+b)}(-1)^{k-1} a^{k-1.5}}{\sigma_{1}}
$$

where $K_{0}=\sigma_{1} \sqrt{\pi(a-b)}$ and the parameters $a, b$, and $\sigma_{1}$ are shown in Figure 1(b).

\section{Boundary Collocation Procedure}

From (14a) and (14b), one can find that dimensionless SIFs can be obtained once the coefficients of $E_{k}$ are determined. In this study, boundary collocation method (BCM), which is a simple and accurate numerical method, is employed to obtain the coefficients of $E_{k}$. In the computational procedure, boundary conditions on the outer boundary are enforced at every collocation point. Both stress and displacement boundary conditions, that is, (6) and (7), are employed for the mixed boundary problem. BCM is applied to form simultaneous equations based on (10a) and (10b) and (11a) and (11b) according to the procedures as follows: first, $N$ collocation points are distributed on the whole boundary with equal distance. Secondly, two coefficient equations will be generated for each point using (10a) and (10b) or (11a) and (11b) depending on the boundary condition. Thus, $2 \mathrm{~N}$ equations can be obtained and expressed in matrix form as

$$
[A]_{2 N \times 4 M}\{X\}_{4 M}=\{F\}_{2 N}
$$

where each element of $[A]_{2 N \times 4 M}$ is the summation of $S_{i k}(i=$ $1,2, \ldots 6)$ and $U_{i k}(i=1,2,3,4)$ that are determined by the coordinates of the collocation points, $\{X\}_{4 M}$ is the unknown coefficient, that is, the real and imaginary parts of $E_{k}$ and $F_{k}$ and $\{F\}_{2 N}$ is the matrix consisting of the accumulative external forces and displacements at the collocation points. Equation (15) can be efficiently solved by means of the least square method, which is the result of Newman's experience [17].

\section{Numerical Results for Cracks under Mixed Boundary Conditions}

Using BCM, the SIF values of the four aforementioned models, that is, a square plate with one crack or with two collinear cracks and an airfoil-shaped plate with one crack or with two collinear cracks, are calculated. Based on the calculation results, the effects of crack orientation, crack distance, and boundary condition on SIF are investigated.

4.1. Effect of Crack Orientations on SIFs for Plates with Single Crack. In order to validate the boundary collocation 


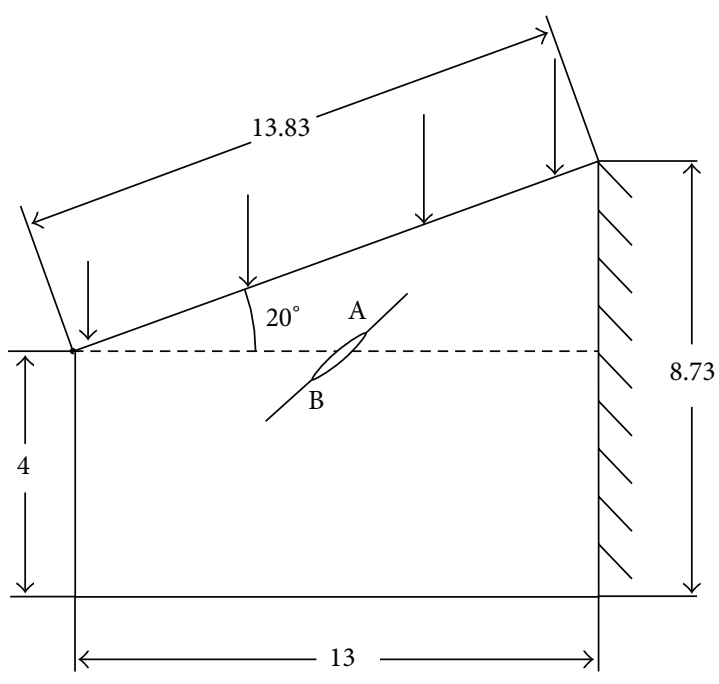

(a) A single crack model

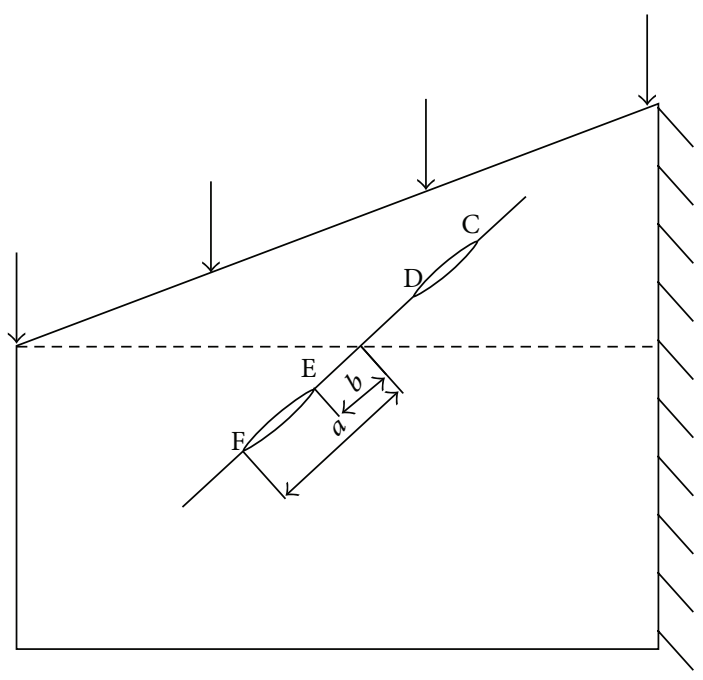

(b) Two collinear cracks model

FIGURE 2: An airfoil-shaped plate with a single crack and two collinear cracks with mixed boundary condition.

method and the calculation procedure, the Mode II stress intensity factors for a square plate with a single crack and an airfoil-shaped plate with single crack under mixed boundary condition as shown in Figures 1(a) and 2(a) are calculated. For each plate, the top boundary is subjected to uniform compressive stress $\sigma_{1}$, while the right boundary is fixed and the others remain stress-free. The collocation points are distributed on the whole boundary of the plate, and some parameters pertinent to the calculation are listed in Tables 1 and 2 , respectively.

Because of two crack tips, the crack is designed to turn from $0^{\circ}$ to $180^{\circ}$ in an anticlockwise sense. As crack tip A turns from $0^{\circ}$ to $180^{\circ}$, tip B will turn from $180^{\circ}$ to $360^{\circ}$. The corresponding Mode II SIFs are calculated and are plotted in Figures 3 and 4 .

From Figure 3, it can be seen that as $\alpha=0^{\circ}$, SIF is larger than 0 . This is because the right edge is fixed and the left edge is stress-free, and the normal compression from the top edge creates an anticlockwise moment, which, similar to the situation in a double cantilever beam subjected to vertical load, forces the upper horizontal crack surface to slide towards the right with respect to the lower crack surface, resulting in a positive shearing SIF value. Both curves first increase and then decrease, and finally they increase again. The SIF curves reach their peak values both about at $\alpha=30^{\circ}$ and then decrease to the minimum values about at $\alpha=118^{\circ}$. The crack orientation at the peak value is called the most unfavourable orientation. Before the peak values, that is, as $\alpha<30^{\circ}$, the load-induced moment plays the predominant role in the determination of crack SIF. As the angles increase to a certain value, the vertical load compels the upper bulk, to which the upper surface is attached, to slide downwards, resulting in a negative SIF value.

The SIF curves for the crack of the airfoil-shaped plate, shown in Figure 4, possess curvilinear trend analogous to those in Figure 3. The SIF of tip A acquires its maximum value
TABLE 1: Calculation parameters for the square plate.

\begin{tabular}{lc}
\hline Crack length & $a-b=0.25$ \\
Crack tip Distance & $2 b=0.125$ \\
Number of summation term & $M=15$ \\
Total number of collocation point & $N=256$ \\
\hline
\end{tabular}

TABLE 2: Calculation parameters for the airfoil-shaped plate.

\begin{tabular}{lc}
\hline Crack length & $a-b=0.25$ \\
Crack tip distance & $2 b=0.125$ \\
Swept back angle & $\alpha=20^{\circ}$ \\
Number of summation term & $M=15$ \\
Total number of collocation point & $N=384$ \\
\hline
\end{tabular}

2.23 at angle $20.8^{\circ}$ and minimum value -2.27 at angle $107.6^{\circ}$. The SIF of tip B reaches its maximum value 2.25 at angle $17.6^{\circ}$ and minimum value -2.20 at angle $107.6^{\circ}$.

Evident differences exist if a detailed examination is taken between the two figures. The amplitude of curves for airfoilshaped plate is much larger than that for square plate. Since the crack in the former plate covers shorter distance to the loaded boundary, which greatly affects the stress field in the vicinity of crack tip, the SIFs for airfoil-shaped plate exhibit higher values. For instance, at angle $45^{\circ}$, the SIF at tip A for square plate is 0.28 , whereas for the airfoil-shaped plate it is 1.25, which represents 3.46 times increase in SIF value. At both crack tips, the SIF curves for the airfoil-shaped plate reach their maximum and minimum values prior to those for the square plate.

4.2. Influence of Crack Orientations on SIF for Plates with Two Collinear Cracks. Materials usually contain a large number of cracks or microcracks, and sometimes the cracks are lined 


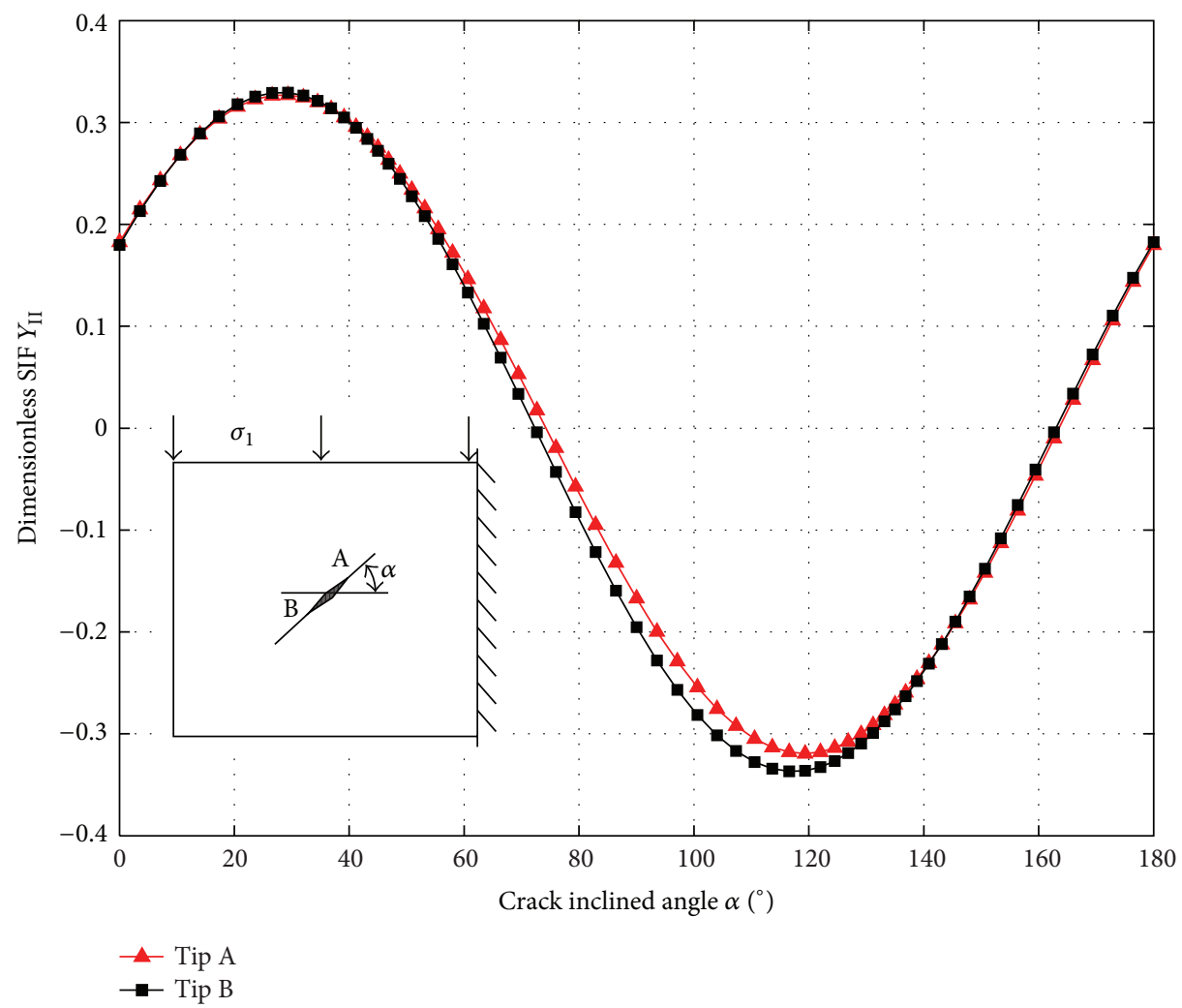

Figure 3: Curves $Y_{\text {II }}{ }^{-} \alpha$ for a mixed boundary square plate with a central crack.

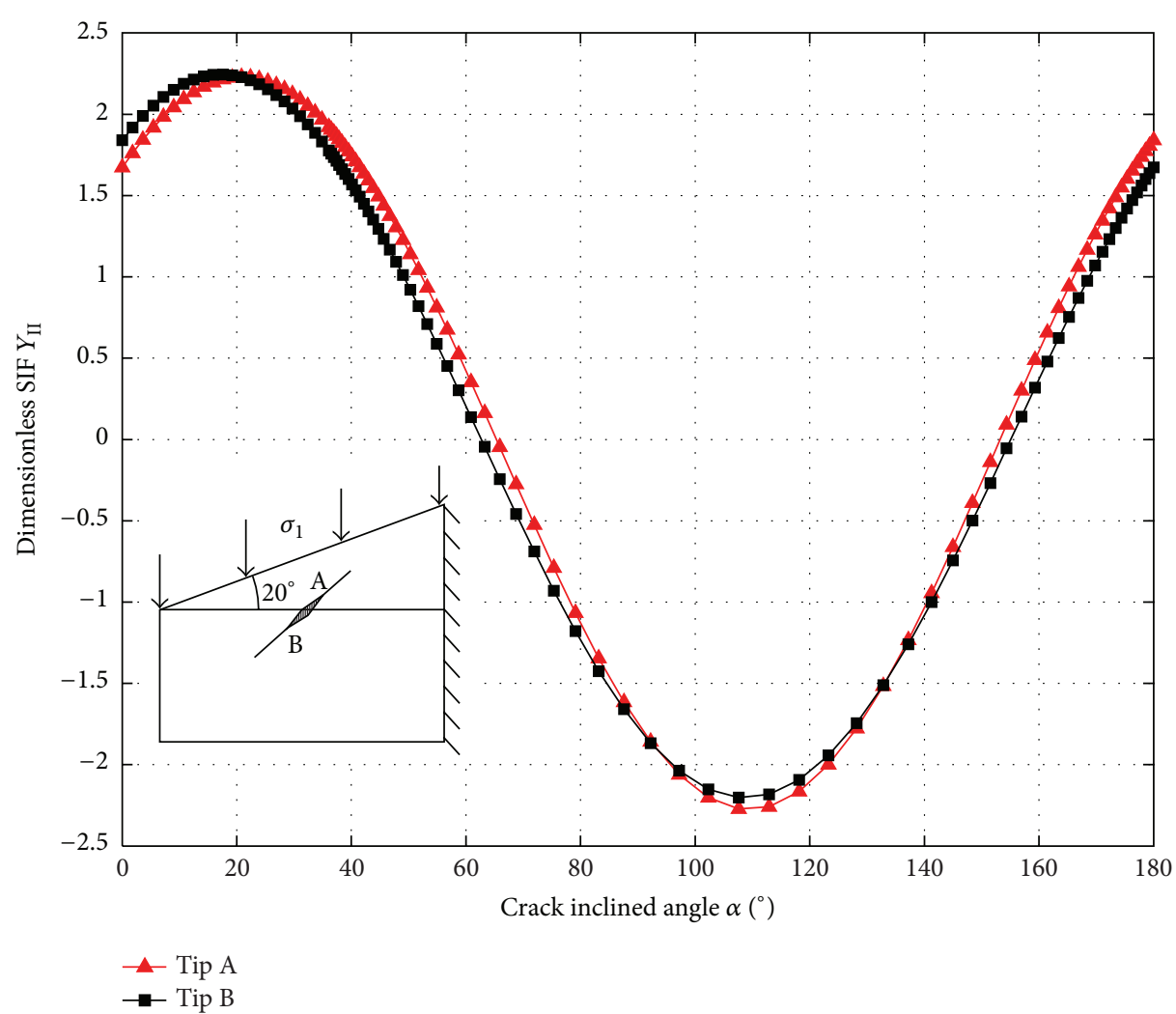

FIGURE 4: Curves $Y_{\text {II }}-\alpha$ for a mixed boundary airfoil-shaped plate with single crack. 


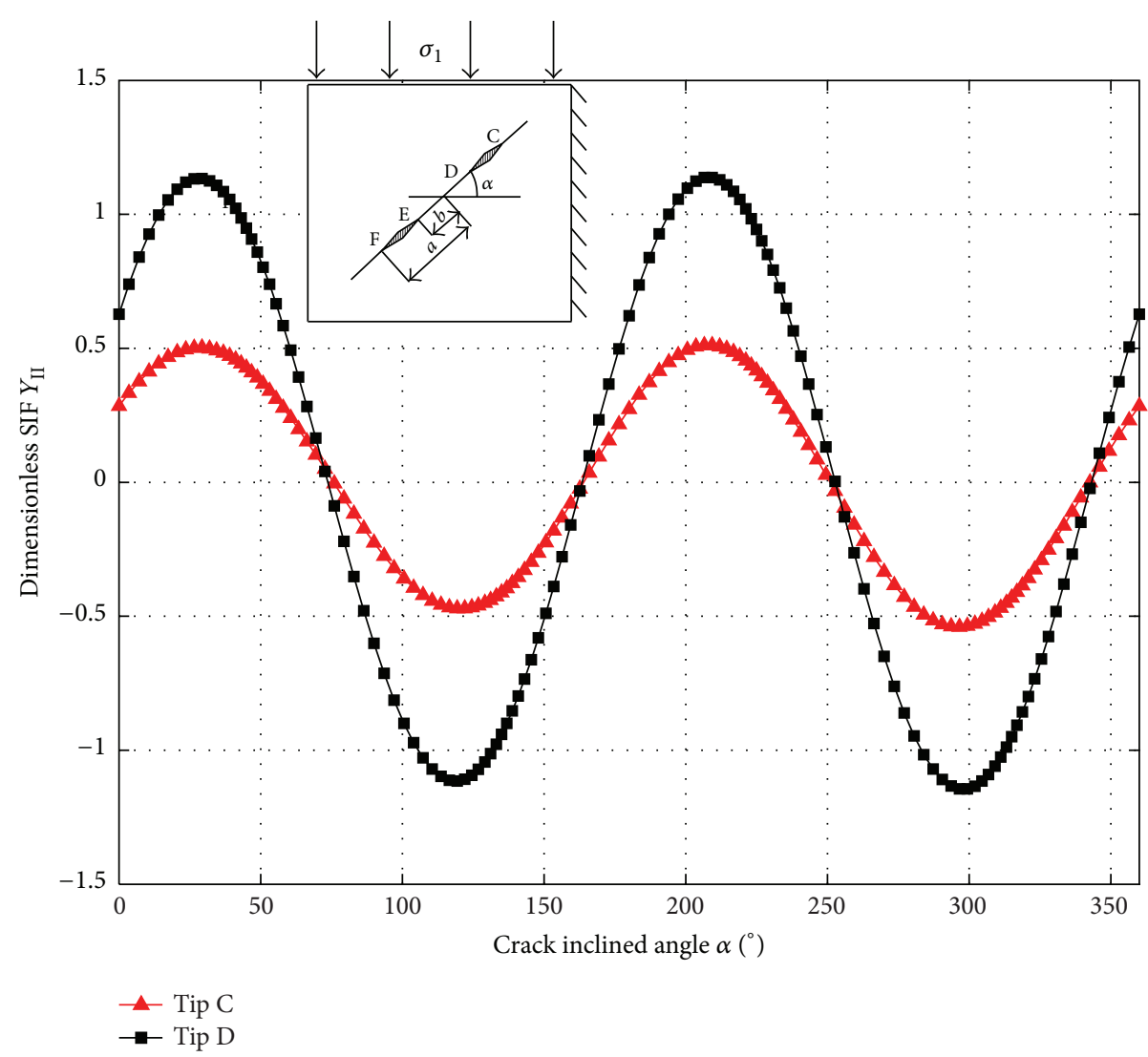

FIGURE 5: Comparison of the $Y_{\text {II }}$ of crack tip C with those of tip D for the square plate.

up, which is called collinear cracks. Figure 1(b) shows a plate with two collinear cracks under mixed boundary conditions. Except for the number of cracks, all other parameters are the same as those shown in Figure 1(a). The corresponding calculation results of the dimensionless SIF $Y_{\text {II }}$ at crack tip C and $\mathrm{D}$ are plotted in Figure 5. It can be seen that the curve amplitude of tip D is larger than that of tip C. This is to be expected, as crack tip D closes to crack tip E, crack tip D is greatly affected by its adjacent crack.

For the airfoil-shaped plate with two collinear cracks as shown in Figure 2(b), the calculation result is shown in Figure 6. Because of the differences in plate shapes and crack locations, the SIF values for airfoil-shaped plate generally are much larger than those of the square plate.

The calculation results show that the difference of SIF values between the two inner crack tips D and E is very small, and it can be easily understood that the nuance of the SIF values at the inner crack tips arises from the asymmetry of stress and displacement boundary conditions.

4.3. Comparison of SIF between One-Crack Model and TwoCrack Model. Brittle materials usually contain a number of cracks, and they affect each other. In this section, the comparison of the SIFs between one-crack model and twocrack model is implemented. For the two-crack model, the distance between two crack tips is 0.125 , which is half of the crack length. The corresponding $Y_{\text {II }}-\alpha$ curves are compared in Figure 7, and it can be found that the $Y_{\mathrm{II}}-\alpha$ curve amplitude for the two-crack model is much larger than that of the one-crack model, which means the more cracks a material possesses, the more unstable the material is.

\subsection{Effect of Crack Distances on SIFs and Experimental Verifi-} cation. In order to investigate the influence of crack distance on SIFs, the square plate with two collinear cracks is employed and the crack orientation is fixed at $30^{\circ}$ which is the most unfavourable orientation shown in Figure 5. The distance $2 b$, between the two crack tips, increases from $0.05(a-b)$ to $3.0(a-b)$, at an interval of $0.05(a-b)$, where $(a-b)$ is half crack length. The corresponding dimensionless SIFs of crack tips $C$ and $D$ are calculated, and the result is shown in Figure 8. When the ratio $2 b /(a-b)$ decreases from 3.0 to 0.5 , the absolute values of SIFs at both tips increase in a slow manner, and as the ratio continues to decrease, the SIF of inner tip D starts to increase rapidly. This means that the effect between two cracks increases as the distance between two cracks reduces.

Photoelastic experiment was carried out to show the characteristics of fringe patterns for varied crack distance and to validate the numerical method introduced in this paper. Four polycarbonate plates with two collinear penetrated cracks were used with the dimension $13 \mathrm{~cm} \times 13 \mathrm{~cm} \times$ $0.6 \mathrm{~cm}$. The crack length is $1.0 \mathrm{~cm}$, and the ratio of crack tip distance versus crack length was set to be $0.0,1.0,2.0$, and 


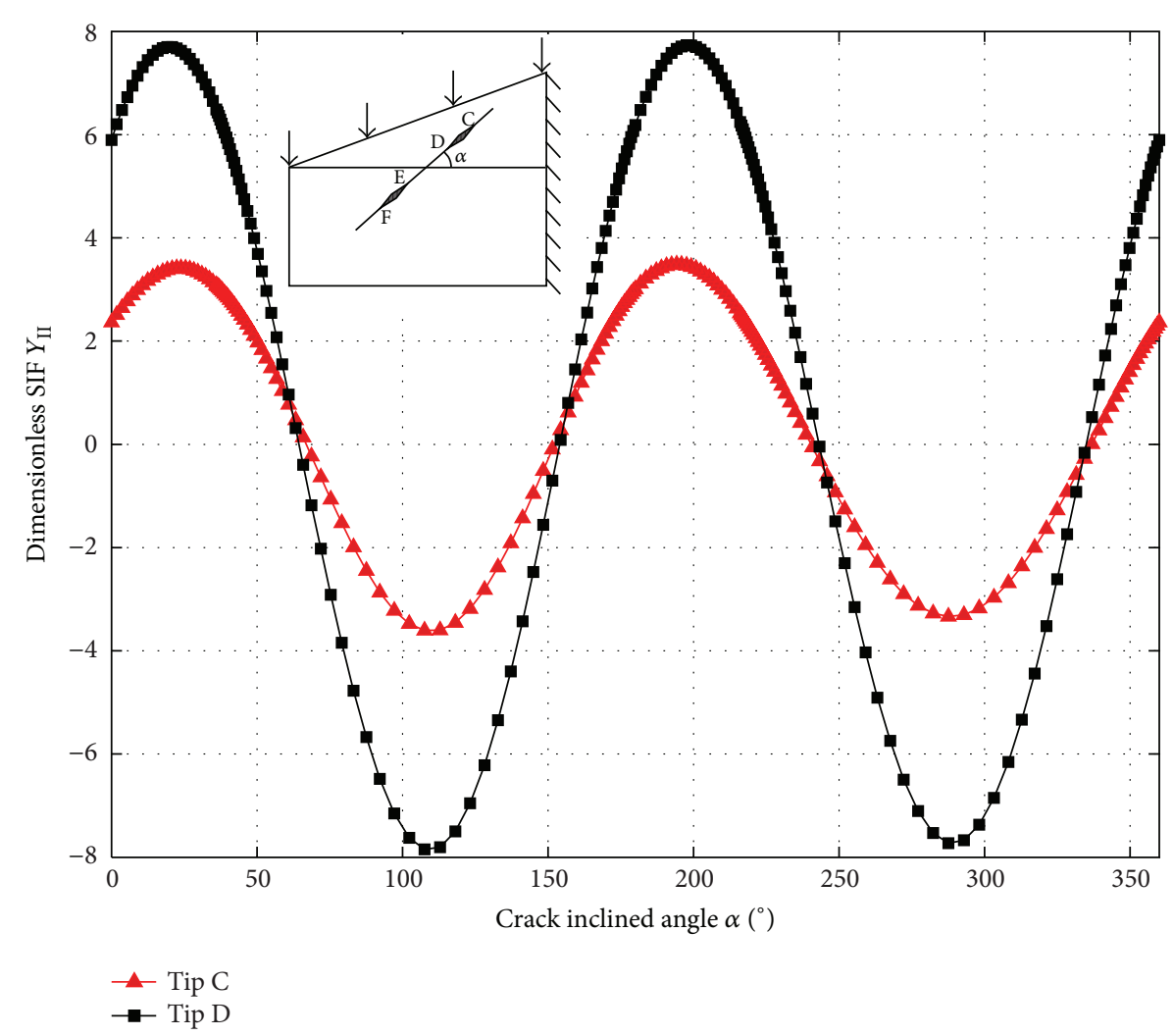

FIgURE 6: Comparison of the $Y_{\text {II }}$ of crack tip C with those of tip D for the airfoil-shaped plate.

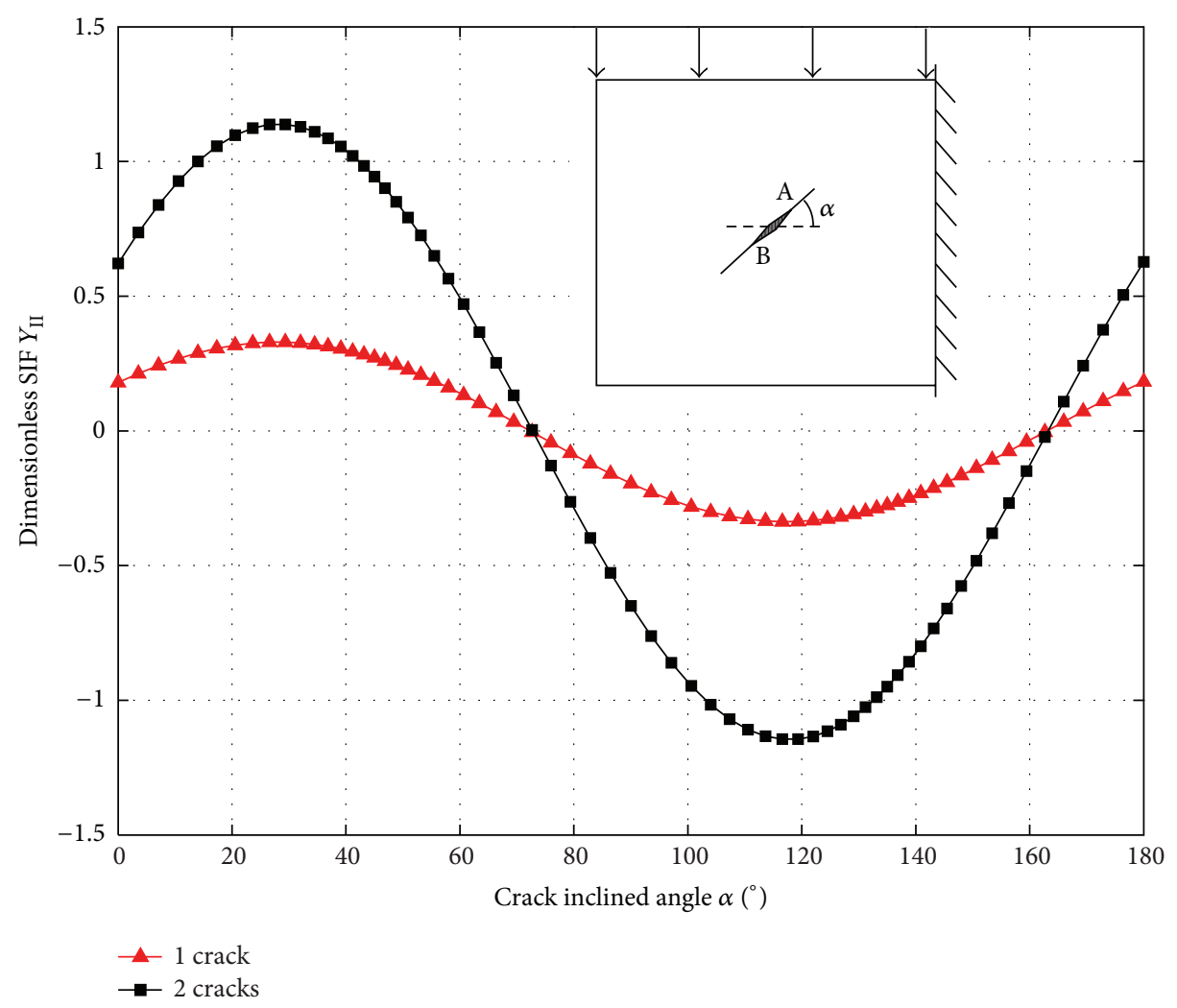

FIGURE 7: SIF comparison for a square plate with one crack and two cracks. 


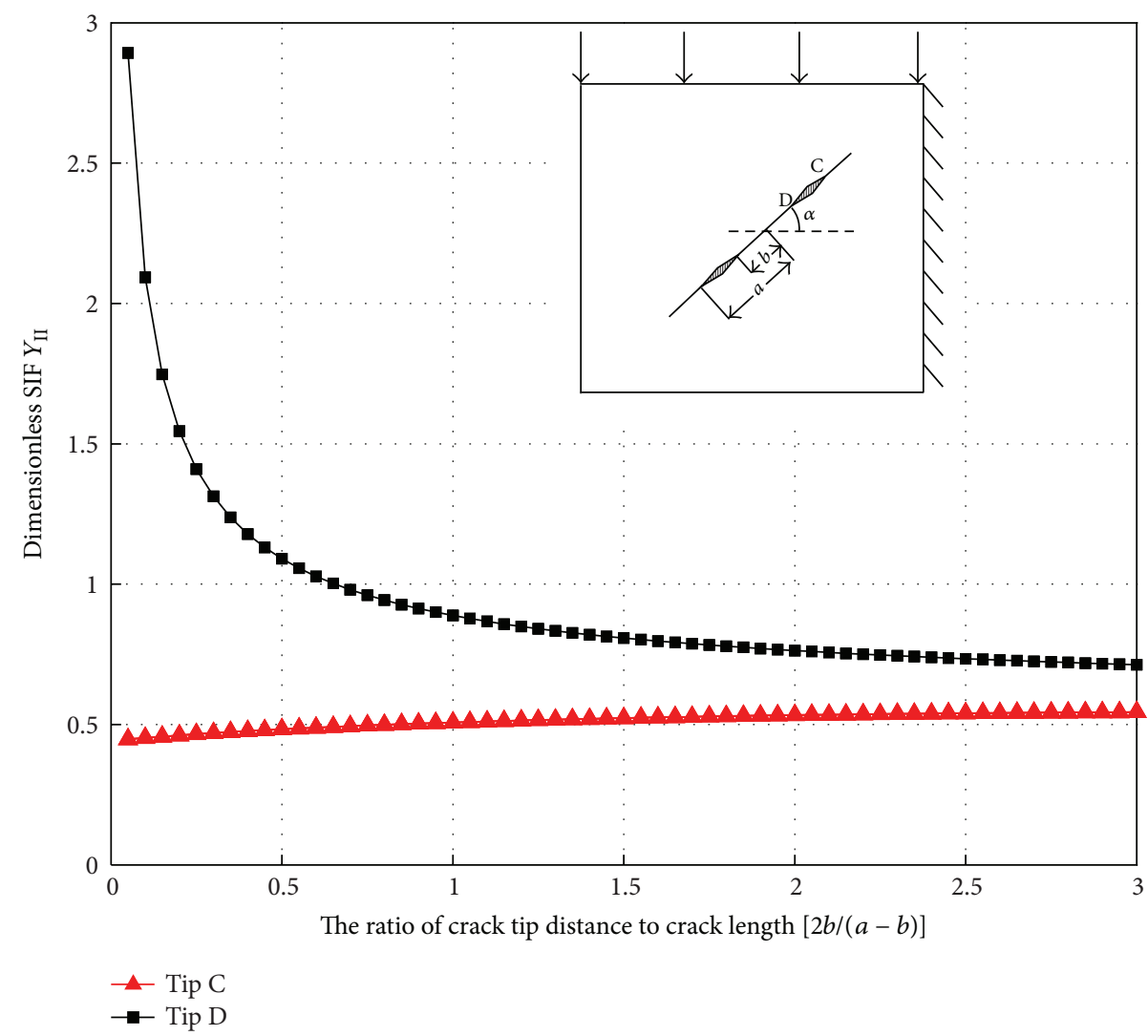

FIGURE 8: Calculation results of $Y_{\text {II }}-2 b /(a-b)$ curves from the square plate model.

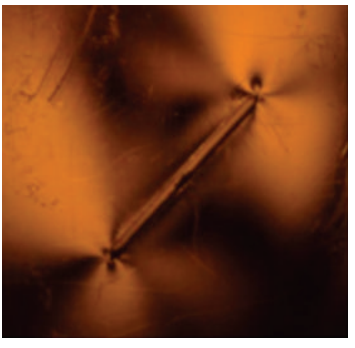

$2 b /(a-b)=0$

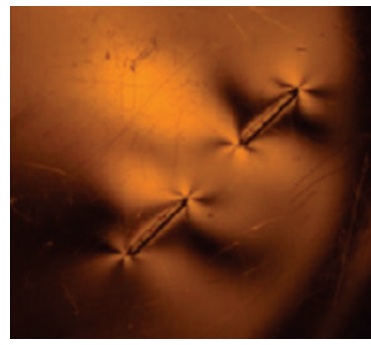

$2 b /(a-b)=1$

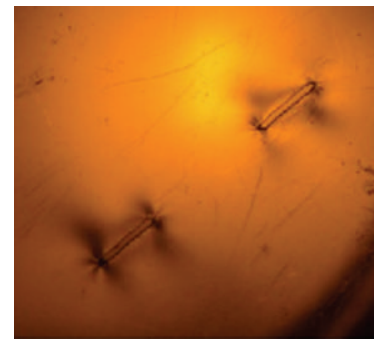

$2 b /(a-b)=2$

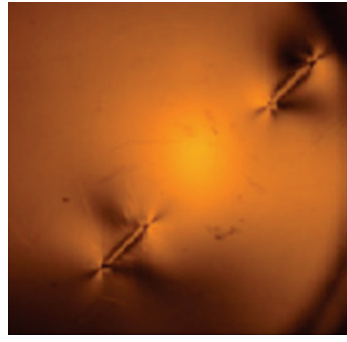

$2 b /(a-b)=3$

FIGURE 9: Photoelastic test result of fringe patterns for the mixed boundary square model with different ratios of crack tip distance to crack length.

3.0, respectively, and the crack orientation was fixed at $45^{\circ}$. Comparing with the numerical results shown in Figure 8, one can find that the photoelastic results shown in Figure 9 generally agree with the numerical result.

4.5. Effect of Boundary Condition. In order to investigate the effect of boundary condition, the corresponding uniaxial compression model of a square plate with two collinear cracks is employed. Except for the boundary, the other parameters are the same as those shown in Figure 1(b). The corresponding calculation results for the models with two different boundary conditions are compared in Figure 10. In order to qualitatively confirm the numerical results, the photoelastic experiment result for the square model under uniaxial compression is presented in Figure 11.

Comparing the fringe patterns shown in Figure 11 with those shown in Figure 9 and combining with the calculation results shown in Figure 10, one can find that the SIFs for the square model under uniaxial compression are about two times larger than those for the square model with mixed boundary conditions, which means that mixed boundaries can affect SIFs largely.

\section{Conclusions}

The stress intensity factors (SIFs) for cracked finite plates with mixed boundary condition are studied in this paper, and a 


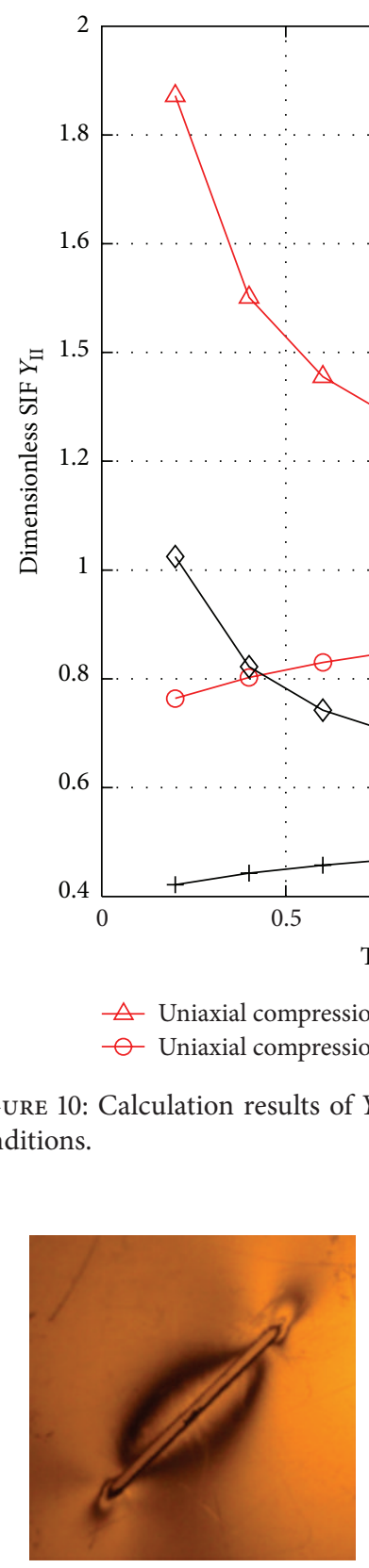

$2 b /(a-b)=0$

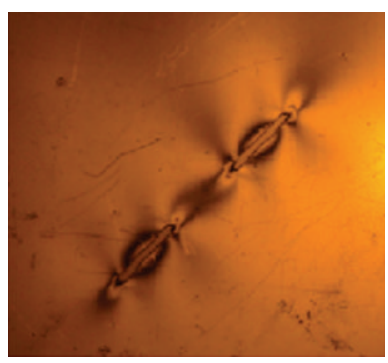

$2 b /(a-b)=1$

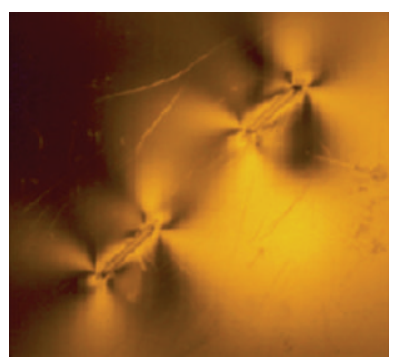

$2 b /(a-b)=2$
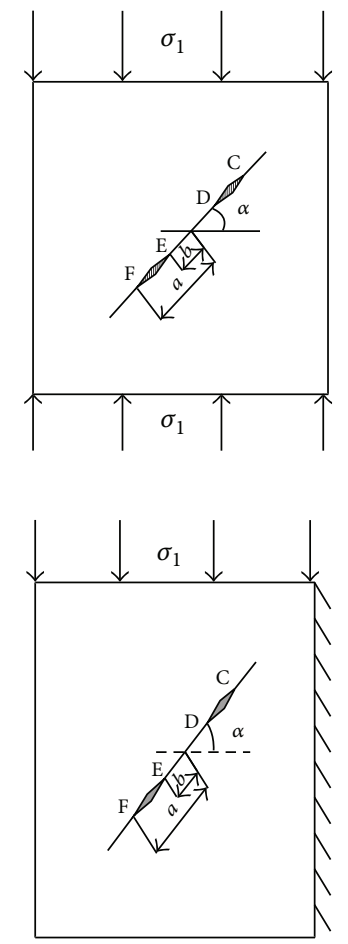

The ratio of crack tip distance to crack length $[2 b /(a-b)]$

$\checkmark$ Mixed boundary Tip D

+ Mixed boundary Tip C

FIGURE 10: Calculation results of $Y_{\mathrm{II}}-2 b /(a-b)$ curves from the square plate model under uniaxial compression and mixed boundary conditions.

FIGURE 11: Photoelastic test result of fringe patterns for the square model under uniaxial compression with different ratios of crack tip distance to crack length.

set of complex stress functions is presented. The unknown coefficients of the stress functions are determined by using boundary collocation method (BCM). Four cases, a square plate containing one-crack or two-cracks and an airfoilshaped plate containing one-crack or two-cracks with mixed boundary condition are investigated, and the corresponding SIFs are calculated. The effects of crack orientation, crack distance, and boundary condition on SIFs are investigated and in order to confirm the calculated results, photoelastic experiments are conducted. From this study, the following conclusions can be obtained.
(1) SIF increases as the distance between two cracks decreases, which means that the effect between cracks increases as the interval distance reduces; in particular as the distance between two crack tips is less than half crack length, the effect between two cracks is intensified largely.

(2) Boundary condition affects SIF significantly. From Figure 10, one can find that for a square plate with two collinear cracks under uniaxial compression, the SIFs are about twice the amount as compared with the mixed boundary as shown in Figure 1(b). 
(3) Comparing with finite element method, BCM has shown its prominent advantages in simplicity of programming, efficiency in saving computer time, and adequate accuracy.

\section{Acknowledgments}

This work was financially supported by the Open Fund of State Key Laboratory of Oil and Gas Reservoir Geology and Exploitation (PLN1202), the National Natural Science Foundation of China (51074109), and by the Major State Basic Research Project (2010CB732005).

\section{References}

[1] G. C. Sih, Handbook of Stress Intensity Factors, Leheigh University, Bethlehem, Pa, USA, 1973.

[2] H. Tada, P. C. Paris, and G. R. Irwin, The Stress Analysis of Cracks Handbook, Del Research Corp, Hellertown, Penn, USA, 1973.

[3] N. I. Muskhelishvili, Some Basic Problems of Mathematical Theory of Elasticity, Noordhoff Press, Amsterdam, The Netherland, 1953.

[4] L. Jing, "A review of techniques, advances and outstanding issues in numerical modelling for rock mechanics and rock engineering," International Journal of Rock Mechanics and Mining Sciences, vol. 40, no. 3, pp. 283-353, 2003.

[5] Y. Murakami, "A simple procedure for the accurate determination of stress intensity factors by finite element method," Engineering Fracture Mechanics, vol. 8, no. 4, pp. 643-655, 1976.

[6] Z. M. Zhu, S. C. Ji, and H. P. Xie, "An improved method of collocation for the problem of crack surface subjected to uniform load," Engineering Fracture Mechanics, vol. 54, no. 5, pp. 731-741, 1996.

[7] Z. Zhu, H. Xie, and S. C. Ji, "The mixed boundary problems for a mixed mode crack in a finite plate," Engineering Fracture Mechanics, vol. 56, no. 5, pp. 647-655, 1997.

[8] Z. Zhu, L. Wang, B. Mohanty, and C. Huang, "Stress intensity factor for a cracked specimen under compression," Engineering Fracture Mechanics, vol. 73, no. 4, pp. 482-489, 2006.

[9] Z. Zhu, "New biaxial failure criterion for brittle materials in compression," Journal of Engineering Mechanics, vol. 125, no. 11, pp. 1251-1258, 1999.

[10] Z. M. Zhu, "Evaluation of the range of horizontal stresses in the earth's upper crust by using a collinear crack model," Journal of Applied Geophysics, vol. 88, pp. 114-121, 2013.

[11] Z. Zhu, Y. Wang, Z. Zhou, B. Li, and H. Xie, "New fracture criterion for brittle materials under compression," Journal of Sichuan University, vol. 40, no. 5, pp. 13-21, 2008.

[12] A. K. Yavuz, S. L. Phoenix, and S. C. TerMaath, "An accurate and fast analysis for strongly interacting multiple crack configurations including kinked (V) and branched (Y) cracks," International Journal of Solids and Structures, vol. 43, no. 22-23, pp. 6727-6750, 2006.

[13] Y. Z. Chen, N. Hasebe, and K. Y. Lee, Multiple Crack Problems in Elasticity, Witpress, Southampton, NY, USA, 2003.

[14] Z. Zhu, "An alternative form of propagation criterion for two collinear cracks under compression," Mathematics and Mechanics of Solids, vol. 14, no. 8, pp. 727-746, 2009.

[15] Y. H. Wang, L. G. Tham, P. K. K. Lee, and Y. Tsui, "A boundary collocation method for cracked plates," Computers and Structures, vol. 81, no. 28-29, pp. 2621-2630, 2003.
[16] W. C. Jin, Z. M. Zhu, and M. Z. Gao, "A general method to determine the stress intensity factor of multiple collinear cracks," Mathematics and Mechanics of Solids, vol. 18, no. 4, pp. 397-408, 2012.

[17] J. C. Newman, "An improved method of collocation for the stress analysis of cracked plate with various shaped boundaries," NASA TN-D-6376, National Aeronautics and Space Administration, Washington, DC, USA, 1971. 

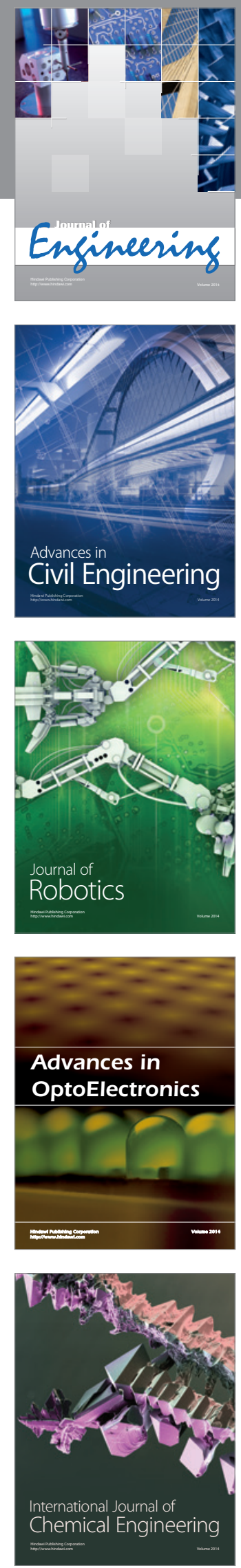

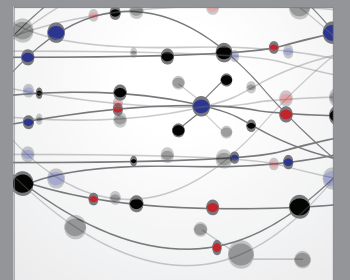

The Scientific World Journal
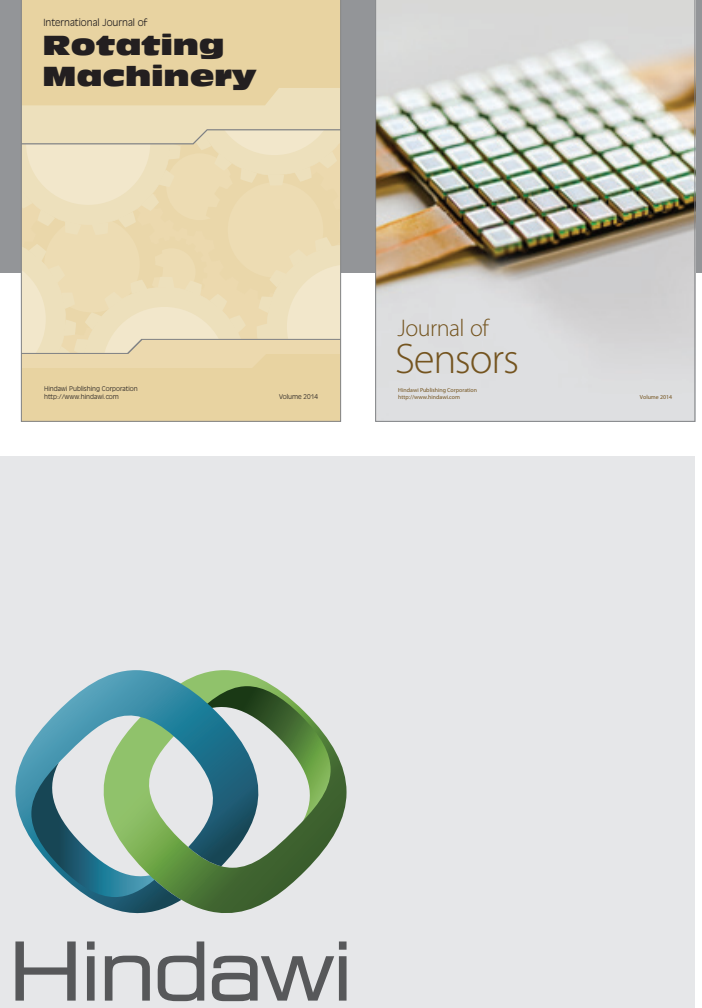

Submit your manuscripts at http://www.hindawi.com
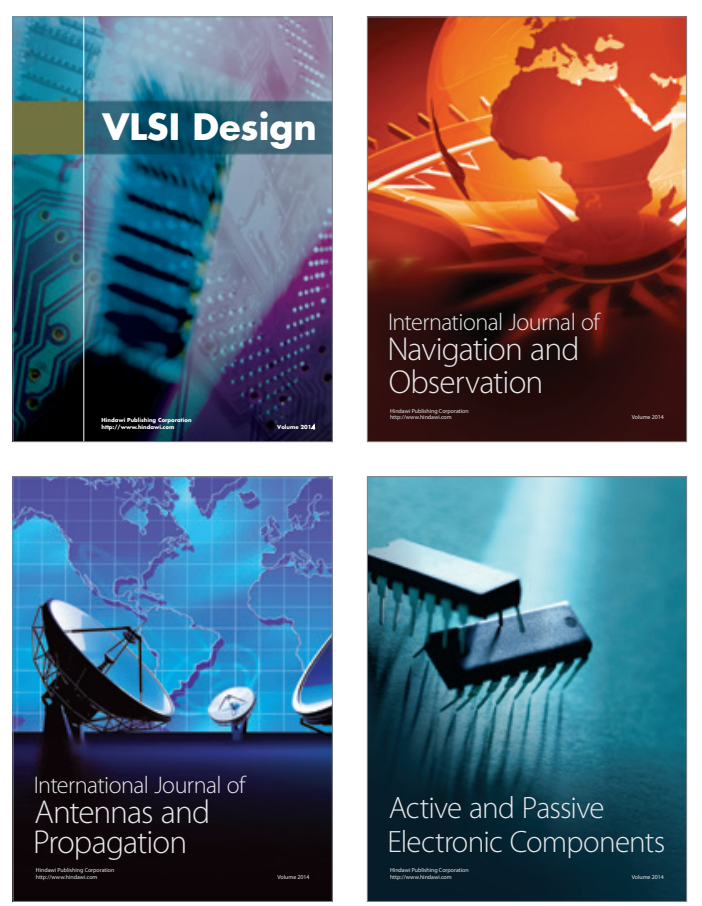
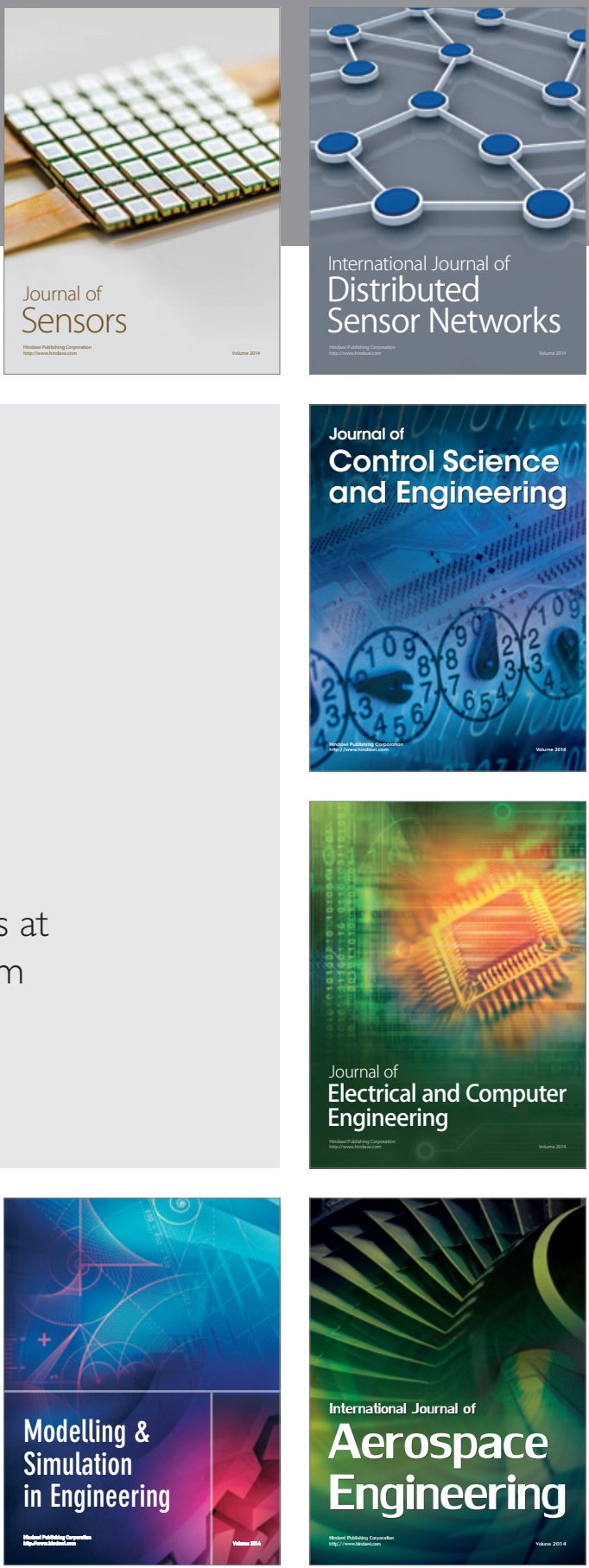

Journal of

Control Science

and Engineering
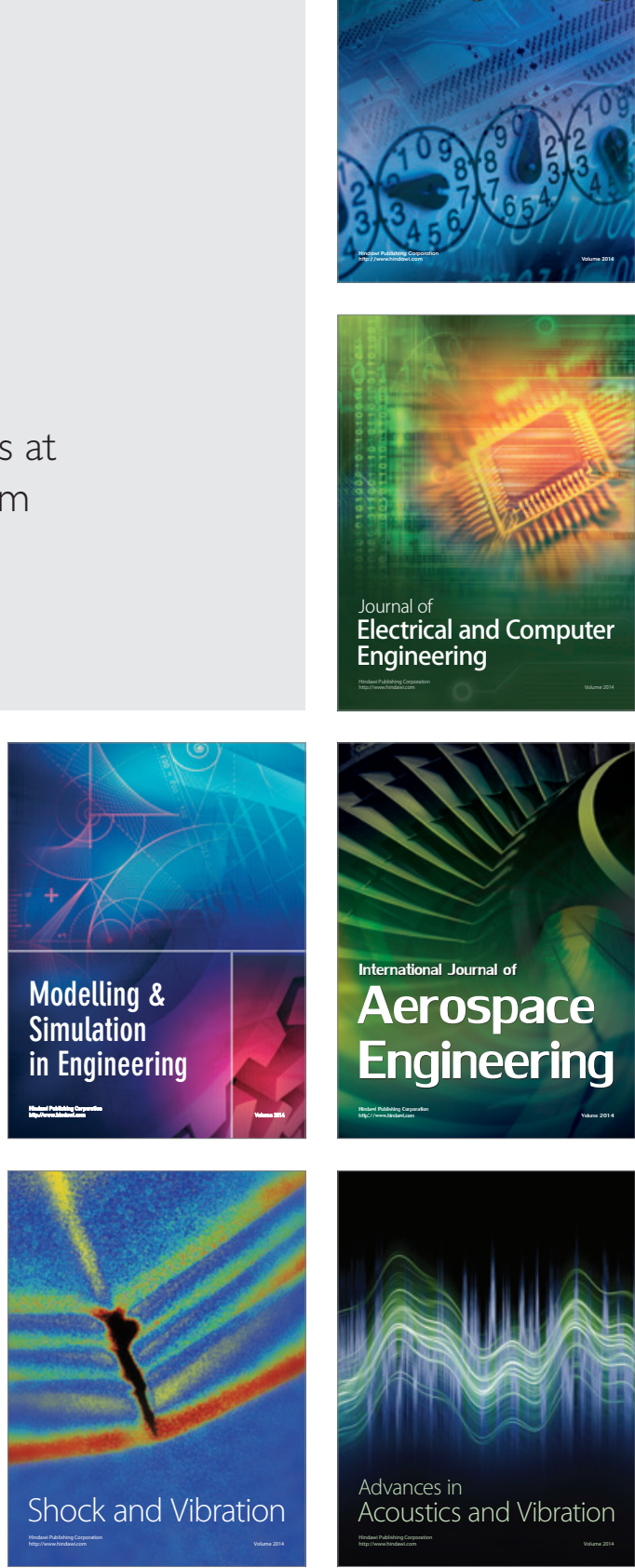